\section{TRANSACTIONS OF THE ROYAL SOCIETY OF TROPICAL MEDICINE AND HYGIENE}

\section{Severe Scorpion Envenomation Among Children: Does Hydrocortisone Improve Outcome? A Case-Control Study}

This paired case-control study examined the safety and efficacy of using hydrocortisone in children with severe scorpion envenomation requiring the intensive care unit (ICU). The study took place in Tunisia, which has an estimated 40,000 scorpion stings per year, with the most severe envenomations caused by the species Androctonus australis and Butus occitanus. The clinical symptoms and signs of scorpion envenomations are caused by massive catecholamine release, direct venom effects, and generalized inflammation. The authors postulate that steroids may reduce the proinflammatory effect of massive cytokine release after scorpion envenomation; however, this is the first study to directly investigate steroid use for scorpion envenomation in children.

The investigators reviewed ICU records for a 13-year period and matched patients receiving steroids with control patients who did not receive steroids by age, severity factors, and scorpion antivenom administration. Primary outcome measures were ICU mortality rate, use of mechanical ventilation, duration of ICU stay, and laboratory analysis. A total of 685 children were admitted for scorpion stings, and 92 received steroids with hydrocortisone hemisuccinate (200-300 mg per 24 hours for 48 hours). The 2 groups had the following similar characteristics: $50 \%$ received the scorpion antivenom, 79\% developed pulmonary edema or cardiogenic shock, and $66 \%$ required catecholamines for hemodynamic support. There was no significant difference in mortality rate $(8 \%)$, the use of mechanical ventilation (35\%), or duration of ICU stay (average 2.6 days) between the 2 groups. The steroid group had a higher rate of hyperglycemia and hypokalemia; however, there were no other significant laboratory differences.

The investigators concluded that the prognosis of children with severe scorpion envenomation admitted to the ICU was not improved by the use of hydrocortisone. Limitations of the study include that is was a single-site investigation and potential changes in clinical management during the study period.

(Trans R Soc Trop Med Hyg. 2013;107:349-355) M Bahloul, A Chaari, R Ammar, et al.

Prepared by Matthew Stewart, MD, University of Utah Emergency Medicine Resident, Salt Lake City, UT, USA.

\section{PUBLIC LIBRARY OF SCIENCE}

\section{Analysis of High-Altitude De-Acclimatization Syndrome After Exposure to High Altitudes: A Cluster-Randomized Controlled Trial}

This randomized cluster-controlled trial investigated high altitude deacclimatization syndrome in 2 groups returning from approximately 6 months of seasonal factory work in Lhasa, China, at an altitude of $3650 \mathrm{~m}$. High altitude deacclimatization syndrome comprises a constellation of symptoms including fatigue, sleepiness, insomnia, memory loss, headache, throat pain, cough, chest tightness, appetite changes, diarrhea, abdominal pain, and arthralgias. The investigators developed a scoring system to evaluate subjects on the 3rd, 50th, and 100th day after descending from high altitude and correlated these scores with laboratory blood tests. Subjects were enrolled from Chongqing, China ( $\mathrm{n}=67,180 \mathrm{~m}$ ) and Kunming, China $(\mathrm{n}=70,1800 \mathrm{~m})$, and matched with healthy control subjects who did not travel to altitude.

In both groups symptom scores peaked on day 3 after returning from high altitude, corresponding to a mild deacclimatization reaction. The most common symptoms of fatigue, dizziness, and coughing were reported in 50 to $73 \%$ of all subjects. Hematologic parameters showed expected patterns, with the hematocrit peaking while at altitude (mean, 57.9\%), and returning to baseline by the 100th day after descent (mean, $43.9 \%$ ). In contrast, white blood cell count, creatine kinase, creatine kinase-MB, and lactate dehydrogenase values peaked on day 3 of return and were higher than high altitude baselines. The authors suggest this finding may be attributable to hypoxia, reoxygenation-induced tissue damage, and the release of inflammatory mediators. The authors proposed their scoring system provided a framework for recognizing high altitude deacclimatization syndrome. Further research is necessary to examine deacclimatization pathophysiology and potential prevention or treatment options. Limitations of the study include a relatively small and underrepresented sample size and the lack of prior studies defining deacclimatization syndrome.

(PLoS One. 2013;8:e62072) B He, J Wang, G Qian, et al. Prepared by Adam Blanchard, MD, University of Utah Department of Emergency Medicine, Salt Lake City, UT, USA.

\section{THE JOURNAL OF STRENGTH AND CONDITIONING RESEARCH}

\section{Differences in Climbing-Specific Strength Between Boulder and Lead Rock Climbers}

Rock climbing is increasingly popular, and the 2 main styles of climbing, leading and bouldering, require different training regimens and physical strength. This cross-sectional case-controlled study investigated grip strength differences among boulder climbers, lead climbers, and nonclimbers. Lead climbing was defined as climbing on higher walls (12-18 m) with the effective climbing time longer when compared with boulder climbing. Bouldering was defined as climbing on lower walls (4-5 m) for shorter amounts of time, generally using multiple attempts to complete the climbing problem.

Although it has been shown that climbers have increased finger flexor maximal strength compared with nonclimbers, there have not been studies among different climbing types. This study compared climbing-specific maximal muscle strength and rapid force capacity of finger flexors between elite boulder and lead climbers. Maximal voluntary contraction 
(MVC) force and rate of force development (RFD) were tested for 2 climbing grips, crimp and open-crimp hand positions. Twenty elite French male rock climbers, 10 bouldering and 10 lead climbers, were matched to control subjects with no rock climbing experience.

Grip strength was measured with an S-shaped strain gauge force transducer with a handmade L-shaped climbing hold. The dominant shoulder and elbow were fully extended, and the force was measured for 3 -second periods for the 2 grip positions. The MVC and RFD among bouldering climbers were stronger than those of lead climbers and nonclimbers. The RFD had the largest difference among the 3 groups with a $38 \%$ difference in open-crimp position and a $34 \%$ difference in the crimp position. The authors proposed that bouldering requires more dynamic and explosive movements in comparison to lead climbing, and this study can help individuals tailor their training for each climbing style. Limitations of the study include a small sample size and possible crossover of finger strength capabilities between bouldering and lead climbing.

(J Strength Cond Res. 2013;27:310-314) M Fanchini, F Violette, FM Impellizzeri, NA Maffiuletti.

Prepared by Wendy Hanna, MD, University of Utah Emergency Medicine Resident, Salt Lake City, UT, USA. 